\title{
EFFICACY OF IVERMECTIN AGAINST GASTROINTESTINAL NEMATODIASIS AND ECTOPARASITES IN CROSSBRED CATTLE IN BANGLADESH
}

\author{
M. Ahammed ${ }^{1}$, M. A. Ali $^{2}$, M. A. Ehsan ${ }^{3^{*}}$ and M. Mostafa ${ }^{1}$ \\ ${ }^{1}$ Department of Pharmacology, Faculty of Veterinary Science, Bangladesh Agricultural University, \\ Mymensingh-2202; ${ }^{2}$ Department of Pathology and Parasitology, Jhenaidah Govt. Veterinary College, Jhenaidah; \\ ${ }^{3}$ Department of Medicine, Faculty of Veterinary Science, Bangladesh Agricultural University, \\ Mymensingh-2202
}

\begin{abstract}
The experiment was carried out to determine the efficacy of Ivermectin (Ivomec ${ }^{\circledR}$ S/C Formulation) against gastrointestinal nematodiasis and ectoparasites including stephanofilaria on crossbred cattle, to determine the effect of drug on certain hematological parameters like hemoglobin $(\mathrm{Hb})$ content, packed cell volume (PCV) and erythrocyte sedimentation rate (ESR)in crossbred cattle and to determine the effect of the drug on live weight. A total of 100 cattle were selected randomly and examined for presence of both endo and ectoparasites including stephanofilaria. Gastrointestinal nematodiasis were detected by examination of fecal samples and ectoparasites as well as stephanofilaria were detected by physical examination. Out of 100 cattle, 20 were found to suffer from both endo and ectoparasites. These 20 cattle were selected finally for the research work. Then these cattle were divided into two groups, group A (treated group, n=15) and group B (control group, $\mathrm{n}=5)$. Ivermectin was injected subcutaneously to the cattle of group A @ $200 \mu \mathrm{m} / \mathrm{kg}$ body weight (1ml/50 kg body weight). The therapeutic efficacy of the drug against gastrointestinal nematodiasis was determined by investigation of fecal egg count reduction and the efficacy was $100 \%$ against the common nematodes in crossbred cattle on day 7, 14, 21 and 28 of post treatment period. Ticks within a markable area (25 square inches) were counted on day 0 and lice infestation was marked as infected on day 0 . Ivermectin showed 100\% effectiveness at the 7, 14, 21 and 28 day of post treatment period against both tick and lice infestation. The efficacy of the drug against stephanofilariasis (humpsore) was determined by reduction of diameter of the sore on the day 14, 42 and 56 of post treatment period. All the sores were completely healed up by the 56 days. During the study of hematological parameters it was seen that $\mathrm{Hb}$ and PCV were increased whereas ESR values were decreased on post treatment days. In this study the mean live weight of the cattle of treated group was increased after treatment with ivermectin and increased body weight was $5.13 \%$ on the 28 th day of post treatment period. From the above findings it may be concluded that ivermectin was $100 \%$ effective against gastrointestinal nematodes common in crossbred cattle. Effectiveness of the drug against external parasites was $100 \%$. In this study hematological parameters were changed significantly after treatment with ivermectin, body weight of animal treated with ivermectin was increased and no adverse effect of the drug was found in this study.
\end{abstract}

Key words: Ivermectin, Gastrointestinal nematodes, ectoparasites, cattle

\section{INTRODUCTION}

Bangladesh, being an agricultural country, is largely dependent on agriculture for its economy. The backbone of agriculture of this country is the livestock but the general condition of this livestock is miserable mainly due to parasitic infestation. Gastrointestinal nematodiasis is a common problem of cattle which causes economic losses in the form of mortality, stunted growth, weight loss, decreased milk and meat production, draft power, market value of living animal, infertility and condemnation of carcasses during meat inspection. In addition to gastrointestinal nematodes, the ectoparasites like tick, mite, lice also cause loss of productivity in cattle. They cause loss of productivity by damaging skin and hides and sucking blood from animal body resulting in poor growth rate of animal. Stephanofilariasis, commonly known as humpsore is very common in Bangladesh and India which causes severe economic losses by decreasing productivity and cost of animals and their skin quality also.

\footnotetext{
*Corresponding e-mail address: amimul.med@bau.edu.bd
} 


\section{Ahammed and others}

In Bangladesh many drugs are being used for a longtime to combat parasitic infestation in livestock. The indiscriminate use of anthelmintics available in the market has made parasites to be resistant against the drugs. Moreover there was not a single anthelmintic effective against both endo and ectoparasites. Among all the anthelmintics in use up to date a very limited number are broad spectrum and effective against a large number of endoparasites. Although there are few ectoparasiticides, they are too toxic for both animal and human being. In this circumstance it was very much urgent to find out an anthelmintic which would be effective against both endo and ectoparasites. This search leads to find out the efficacy of ivermectin against both the types of parasites. There was no available report on efficacy of ivermectin in the crossbred cattle which are more susceptible to parasitic infestations than our indigenous cattle. Considering all these constraints this work aimed to determine the efficacy of ivermectin (subcutaneous injection formulation) against gastrointestinal nematodes and ectoparasites in crossbred cattle along with the effect of Ivermectin on certain blood parameters and body weight of crossbred cattle.

\section{MATERIALS AND METHODS}

The experiment was carried out in two villages namely Kewatkhali and Balashpur and BAU Dairy Farm nearby Bangladesh Agricultural University, Mymensingh. The following procedure was adopted to perform the experiment-

Primarily a total of 100 crossbred cattle were selected randomly and the cattle were examined carefully to detect the presence of both endo and ectoparasites by fecal and physical examination respectively. By fecal examination gastrointestinal nematodiasis were recorded in seventy five cattle. By physical examination it was found that 40 animals had tick infestation, 30 animals had lice infestation and 20 animals had humpsore. Out of the primarily selected 100 crossbred cattle, 20 animals harboring both endo and ectoparasites were finally selected for the present study. These twenty cattle were randomly divided into two groups- group A (treated group) consisting of 15 cattle and group B (control group) consisting of the rest 5 cattle. These selected animals were marked by plating at the neck by wire.

For the detection of gastrointestinal nematodiasis fecal sample was collected directly from the rectum of the cattle under experiment belonging to both group A and group B. It was performed both at pre and post treatment period. After treatment it was done on $7^{\text {th }}, 14^{\text {th }}, 21^{\text {st }}$ and $28^{\text {th }}$ day. The collected samples have been examined by Direct Smear Method and McMaster Egg Counting Technique as described by Soulsby (1986). The presence of ectoparasites on body coat of cattle was detected by thorough physical examination and the infestation was marked as slight, moderate and severe. Tick and lice infestations and stephanophilariasis were considered in this study.

\section{Selection and administration of drug}

Injectable ivermectin preparation (Ivomec S/C Formulation) was purchased from the local market. Then the drug was injected subcutaneously to all the cattle of group A at the dose rate of $1 \mathrm{ml} / 50 \mathrm{~kg}$ body weight $(0.2$ $\mathrm{mg} / \mathrm{kg}$ body weight. The egg reduction test was performed on the $7^{\text {th }}, 14^{\text {th }}, 21^{\text {st }}$ and $28^{\text {th }}$ day of post treatment period. The ticks within a selected area ( 25 square inches) of body coat were counted physically prior to treatment with ivermectin ( day 0 ) and after treatment on the $7^{\text {th }}, 14^{\text {th }}, 21^{\text {st }}$ and $28^{\text {th }}$ day. The total count of ticks was recorded. The cattle infestated with lice were marked as infestated on day 0 . After treatment with ivermectin observations on the basis of physical examination were recorded on the $7^{\text {th }}, 14^{\text {th }}, 21^{\text {st }}$ and $28^{\text {th }}$ day. The diameter of hump sore was recorded before treatment and on the day 14, 28, 42 and 56 after treatment with the drug. To study the effect of ivermectin on hematological parameters, blood samples were collected from the external jugular vein of the cattle of treated and control group in vials containing anticoagulant (Sodium EDTA) at the day $0,7,14,21$ and 28 to determine the effect of Ivermectin to the following hematological parametershemoglobin ( $\mathrm{Hb})$ content, packed cell volume (PCV) and erythrocyte sedimentation rate (ESR). The hematological parameters were determined as per standard method cited by Coffin (1955)

\section{Statistical Analysis}

The data were analyzed statistically between pretreatment and post treatment values by using well known student ' $\mathrm{T}$ ' test. 
Efficacy of ivermectin in crossbred cattle

\section{RESULTS AND DISCUSSION}

The cattle belonging to the group A were treated with Ivermectin at the dose rate of $1 \mathrm{ml}$ per $50 \mathrm{~kg}$ body weight $\left(200 \mu \mathrm{g} /\right.$ per kg body weight) and there gastrointestinal nematodiasis showed $100 \%$ egg reduction on the $7^{\text {th }}$ day of the post treatment period. The same result was found on the $14^{\text {th }}, 21^{\text {st }}$ and $28^{\text {th }}$ day of post treatment period. In control group (Group B) the EPG was increased gradually on the $7^{\text {th }}, 14^{\text {th }}, 21^{\text {st }}$ and $28^{\text {th }}$ day (Table 1 ).

Table 1. Efficacy of Ivermectin (S/C formulation) against gastrointestinal nematodiasis based on the number of egg in feces

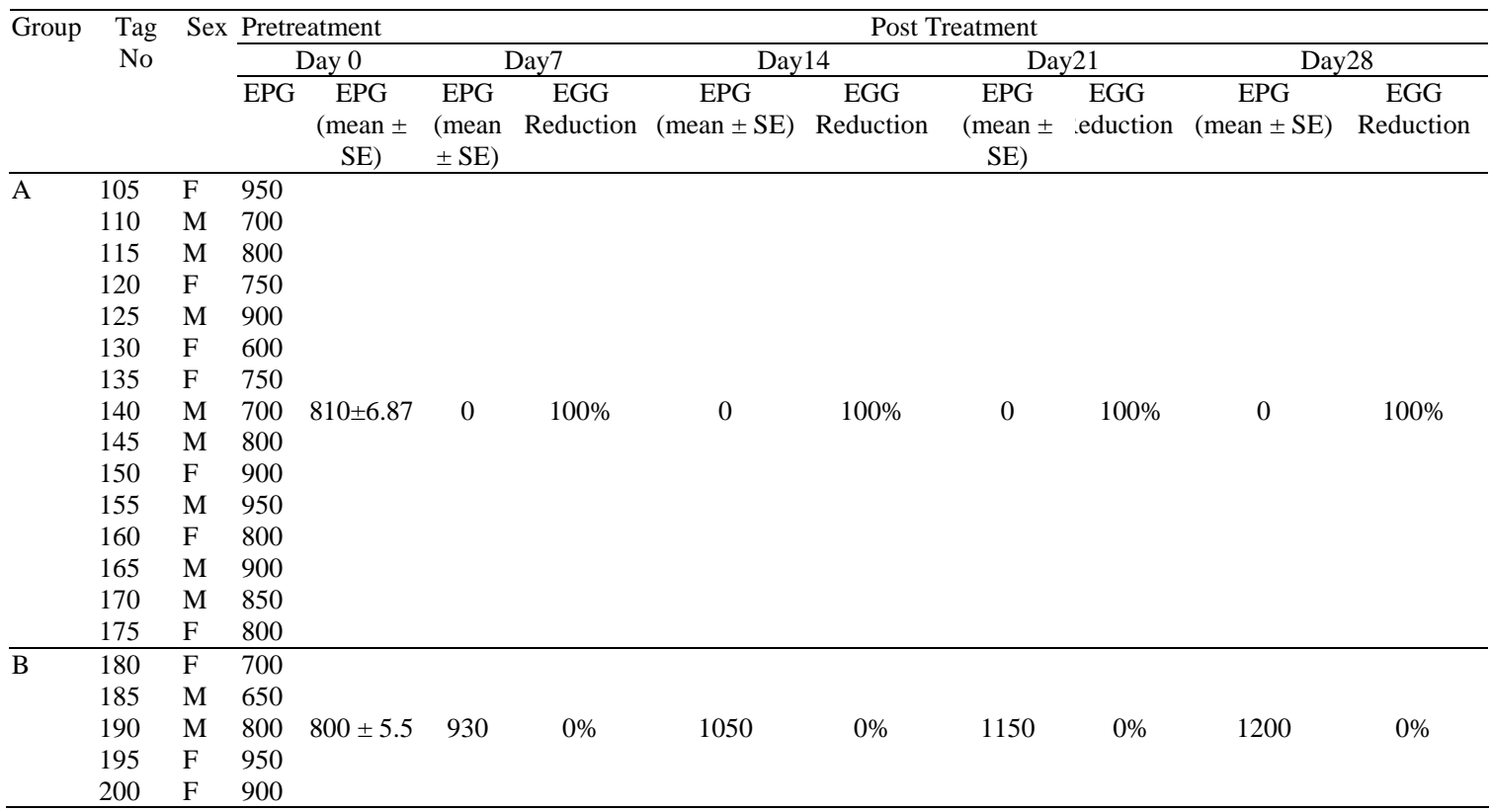

Ivermectin showed 100\% efficacy against lice infestation of Group A cattle which were treated with the drug at the dose rate of $1 \mathrm{ml} / 50 \mathrm{~kg}$ body weight $(200 \mu \mathrm{g} / \mathrm{kg}$ body weight). No lice was found by physical examination on the $7^{\text {th }}$ day of treatment. The similar result was found on the $14^{\text {th }} 21^{\text {st }} \& 28^{\text {th }}$ day of post treatment period. Whereas the rate of lice infestation was constant in the cattle of group B (Control Group) this finding was shown in Table 2.

The number of ticks of cattle of group A were determine by physical examination within a selected area (25 square inches). Again ticks in the selected area were counted on the $7^{\text {th, }} 14^{\text {th, }} 21^{\text {st }}$ and $26^{\text {th }}$ day after treatment with ivermectin. No ticks were found with the selected area of the body of cattle. On the other hand in control group the numbers of ticks were increased gradually on the $7^{\text {th }}, 14^{\text {th }}, 21^{\text {st }} \& 28^{\text {th }}$ day (Table 3 ).

The cattle of Group A with humpsore showed significant reduction of lesion 14 days after a single dose of Ivermectin( S/C Formulation). After $42^{\text {nd }}$ day of post treatment period smaller lesions were completely healed up but the larger lesions were not completely healed. By the $56^{\text {th }}$ day of post treatment period the larger lesions were also healed up completely leaving the scar tissue. So it can be stated that S/C injection of Ivermectin (Ivomec ${ }^{\circledR}$ ) is very much effective drug against stephanofilariasis for crossbred cattle in Bangladesh.

\section{Effect of Ivermectin (S/C Formulation) on certain Hematological Parameters (Hb, PCV and ESR)}

Although no significant change in $\mathrm{gm} \%$ of $\mathrm{Hb}$ was observed at the $7^{\text {th }}$ day of post treatment period, it was increased significantly from the $14^{\text {th }}$ day onwards and continued upto $28^{\text {th }}$ day of treatment. No such change was observed in cattle of control group (Table 4). 


\section{Ahammed and others}

Table 2. Efficacy of Ivermectin (S/C Formulation) against lice infestation in crossbred cattle

\begin{tabular}{|c|c|c|c|c|c|c|c|c|c|c|c|}
\hline \multirow[t]{2}{*}{ Group } & \multirow{2}{*}{$\begin{array}{l}\text { No of } \\
\text { cattle }\end{array}$} & \multirow[t]{2}{*}{ Drug with dose } & \multirow{2}{*}{$\begin{array}{c}\text { Pretreatment } \\
\text { day }\end{array}$} & \multicolumn{2}{|c|}{$7^{\text {th }}$ Day } & \multicolumn{2}{|c|}{$14^{\text {th }}$ Day } & \multicolumn{2}{|c|}{$21^{\text {st }}$ Day } & \multicolumn{2}{|c|}{$28^{\text {th }}$ Day } \\
\hline & & & & Findings & $\begin{array}{c}\% \\
\text { Reduction }\end{array}$ & Findings & $\begin{array}{c}\% \\
\text { Reduction }\end{array}$ & Findings & $\begin{array}{c}\% \\
\text { Reduction }\end{array}$ & Findings & $\begin{array}{c}\% \\
\text { Reduction }\end{array}$ \\
\hline $\bar{A}$ & 15 &  & Infected & Nil & $100 \%$ & Nil & $100 \%$ & Nil & $100 \%$ & Nil & $100 \%$ \\
\hline B & 5 & Nil & Infected & Infected & 0 & Infected & 0 & Infected & 0 & Infected & 0 \\
\hline
\end{tabular}

Table 3. Efficacy of Ivermectin (S/C formulation) against ticks infestation in crossbred cattle

\begin{tabular}{|c|c|c|c|c|c|c|c|c|c|c|c|c|c|}
\hline \multirow[t]{3}{*}{ Group } & \multirow{3}{*}{$\begin{array}{l}\text { Tag } \\
\text { No }\end{array}$} & \multirow[t]{3}{*}{ Sex } & \multirow{3}{*}{$\begin{array}{l}\text { Drug with } \\
\text { dose }\end{array}$} & \multirow{2}{*}{\multicolumn{2}{|c|}{ Pretreatment }} & \multicolumn{8}{|c|}{ Post treatment } \\
\hline & & & & & & \multicolumn{2}{|c|}{$7^{\text {th }}$ Day } & \multicolumn{2}{|c|}{$14^{\text {th }}$ Day } & \multicolumn{2}{|c|}{$21^{\text {st }}$ Day } & \multicolumn{2}{|c|}{$28^{\text {th }}$ Day } \\
\hline & & & & $\begin{array}{l}\text { Number of } \\
\text { ticks within } \\
25 \text { sq,inches }\end{array}$ & $\begin{array}{c}\text { Mean } \pm \\
\text { SE }\end{array}$ & $\begin{array}{l}\text { Number } \\
\text { of ticks } \\
\text { (mean) }\end{array}$ & $\begin{array}{c}\% \\
\text { Reduction }\end{array}$ & $\begin{array}{l}\text { Number } \\
\text { of ticks } \\
\text { (mean) }\end{array}$ & $\begin{array}{c}\% \\
\text { Reduction }\end{array}$ & $\begin{array}{l}\text { Number } \\
\text { of ticks } \\
\text { (mean) }\end{array}$ & $\begin{array}{c}\% \\
\text { Reduction }\end{array}$ & $\begin{array}{c}\text { Number } \\
\text { of ticks } \\
\text { (mean) }\end{array}$ & $\begin{array}{c}\% \\
\text { Reduction }\end{array}$ \\
\hline \multirow[t]{15}{*}{ A } & 105 & $\mathrm{~F}$ & Ivermectin & 7 & & & & & & & & & \\
\hline & 110 & M & @ & 9 & & & & & & & & & \\
\hline & 115 & M & $200 \mu \mathrm{gm} /$ & 8 & & & & & & & & & \\
\hline & 120 & $\mathrm{~F}$ & $\mathrm{~kg}$ & 10 & & & & & & & & & \\
\hline & 125 & M & body & 8 & & & & & & & & & \\
\hline & 130 & $\mathrm{~F}$ & weight & 7 & & & & & & & & & \\
\hline & 135 & $\mathrm{~F}$ & & 9 & & & & & & & & & \\
\hline & 140 & M & & 10 & $7.0 \pm 0.45$ & 0 & $100 \%$ & 0 & $100 \%$ & 0 & $100 \%$ & 0 & $100 \%$ \\
\hline & 145 & M & & 7 & & & & & & & & & \\
\hline & 150 & $\mathrm{~F}$ & & 9 & & & & & & & & & \\
\hline & 155 & M & & 8 & & & & & & & & & \\
\hline & 160 & $\mathrm{~F}$ & & 10 & & & & & & & & & \\
\hline & 165 & M & & 6 & & & & & & & & & \\
\hline & 170 & M & & 7 & & & & & & & & & \\
\hline & 175 & $\mathrm{~F}$ & & 4 & & & & & & & & & \\
\hline \multirow[t]{5}{*}{ B } & 180 & $\mathrm{~F}$ & & 5 & & & & & & & & & \\
\hline & 185 & M & $\mathrm{Nil}$ & 9 & & & & & & & & & \\
\hline & 190 & M F & & 8 & $7.8 \pm 1.98$ & 8.8 & 0 & 10 & 0 & 11.5 & 0 & 12.5 & 0 \\
\hline & 195 & $\mathrm{~F}$ & & 10 & & & & & & & & & \\
\hline & 200 & & & 7 & & & & & & & & & \\
\hline
\end{tabular}

Table 4.Efficacy of Ivermectin (S/C Formulation) on Hemoglobin (Hb gm\%) in crossbred cattle.

\begin{tabular}{|c|c|c|c|c|c|c|c|c|c|c|c|c|}
\hline \multirow[t]{3}{*}{ Group } & \multirow{3}{*}{$\begin{array}{l}\text { Tag } \\
\text { No }\end{array}$} & \multirow{3}{*}{$\begin{array}{l}\text { Drug with } \\
\text { dose }\end{array}$} & \multirow{2}{*}{\multicolumn{2}{|c|}{ Pretreatment 0 Day }} & \multicolumn{8}{|c|}{ Post Treatment } \\
\hline & & & & & \multicolumn{2}{|c|}{$7^{\text {th }}$ Day } & \multicolumn{2}{|c|}{$14^{\text {th }}$ Day } & \multicolumn{2}{|c|}{$21^{\text {st }}$ Day } & \multicolumn{2}{|c|}{$28^{\text {th }}$ Day } \\
\hline & & & $\begin{array}{c}\mathrm{Hb} \text { in } \\
\text { individual } \\
\text { animal }\end{array}$ & Mean \pm SE & $\begin{array}{c}\mathrm{Hb} \text { in } \\
\text { individual } \\
\text { animal }\end{array}$ & Mean \pm SE & $\begin{array}{c}\mathrm{Hb} \text { in } \\
\text { individual } \\
\text { animal }\end{array}$ & Mean \pm SE & $\begin{array}{c}\mathrm{Hb} \text { in } \\
\text { individual } \\
\text { animal }\end{array}$ & Mean \pm SE & $\begin{array}{c}\text { Hb in } \\
\text { individual } \\
\text { animal }\end{array}$ & $\begin{array}{c}\text { Mean } \\
\pm \text { SE }\end{array}$ \\
\hline \multirow[t]{15}{*}{ A } & 105 & Ivermectin & 6.5 & & 7.0 & & 7.5 & & 7.8 & & 8.0 & \\
\hline & 110 & @ & 8.0 & & 8.4 & & 8.5 & & 9.0 & & 9.5 & \\
\hline & 115 & $200 \mu \mathrm{g} / \mathrm{kg}$ & 7.5 & & 8.0 & & 8.5 & & 8.8 & & 9.0 & \\
\hline & 120 & body & 8.5 & & 8.56 .8 & & 9.0 & & 9.5 & & 10.0 & \\
\hline & 125 & weight & 6.0 & & 9.5 & & 7.0 & & 7.8 & & 8.5 & \\
\hline & 130 & & 9.0 & & 8.0 & & 9.8 & & 10.0 & & 10.0 & \\
\hline & 135 & & 7.5 & & 9.0 & & 8.5 & & 9.0 & & 9.8 & \\
\hline & 140 & & 8.5 & & 7.0 & & 9.4 & & 9.5 & & 10.0 & \\
\hline & 145 & & 6.5 & $7.53 \pm 0.26$ & 7.0 & $7.98 \pm 0.25$ & 7.5 & $8.40 \pm 0.22$ & 8.0 & $8.94 \pm 0.214$ & 8.8 & $9.48 \pm 0.21$ \\
\hline & 150 & & 6.5 & & 8.0 & & 7.8 & & 8.4 & & 9.0 & \\
\hline & 155 & & 7.5 & & 9.5 & & 8.5 & & 8.8 & & 9.5 & \\
\hline & 160 & & 9.0 & & 8.5 & & 9.5 & & 10.0 & & 10.5 & \\
\hline & 165 & & 8.0 & & 8.7 & & 9.0 & & 10.0 & & 10.4 & \\
\hline & 170 & & 7.5 & & 6.8 & & 8.0 & & 9.5 & & 10.0 & \\
\hline & 175 & & 6.5 & & & & 7.5 & & 8.0 & & 8.8 & \\
\hline \multirow[t]{5}{*}{$\mathrm{B}$} & 180 & & 7.5 & & 7.5 & & 7.0 & & 6.8 & & 6.5 & \\
\hline & 185 & & 8.0 & $7.36 \pm 0.30$ & 8.0 & & 7.8 & & 7.0 & & 6.5 & \\
\hline & 190 & $\mathrm{Nil}$ & 7.8 & & 7.5 & $7.30 \pm 0.29$ & 7.5 & $7.10 \pm 0.28$ & 7.4 & $6.74 \pm 0.26$ & 7.0 & \\
\hline & 195 & & 6.5 & & 6.5 & & 6.4 & & 6.0 & & 5.8 & $6.36 \pm 0.24$ \\
\hline & 200 & & 7.0 & & 7.0 & & 6.8 & & 6.5 & & 6.0 & \\
\hline
\end{tabular}


PCV values of treatment group increased significantly at the $28^{\text {th }}$ day of treatment but it was decreased in control group. These findings of PCV values were shown in Table 5.

Table 5. Efficacy of Ivermectin (S/C Formulation) on Packed Cell Volume (PCV\%) in crossbred cattle

\begin{tabular}{|c|c|c|c|c|c|c|c|c|c|c|c|c|}
\hline \multirow[t]{3}{*}{ Group } & \multirow[t]{3}{*}{$\begin{array}{l}\text { Tag } \\
\text { No }\end{array}$} & \multirow{3}{*}{$\begin{array}{l}\text { Drug } \\
\text { with } \\
\text { dose }\end{array}$} & \multicolumn{2}{|c|}{$\begin{array}{l}\text { Pretreatment } \\
\text { Day 0 }\end{array}$} & \multicolumn{8}{|c|}{ Post Treatment } \\
\hline & & & \multirow{2}{*}{$\begin{array}{l}\text { PCV in } \\
\text { individua } \\
\text { l cattle }\end{array}$} & \multirow[t]{2}{*}{ Mean \pm SE } & \multicolumn{2}{|c|}{$7^{\text {th }}$ day } & \multicolumn{2}{|c|}{$14^{\text {th }}$ day } & \multicolumn{2}{|c|}{$21^{\text {st }}$ day } & \multicolumn{2}{|c|}{$28^{\text {th }}$ day } \\
\hline & & & & & $\begin{array}{c}\text { PCV in } \\
\text { individual } \\
\text { cattle }\end{array}$ & Mean \pm SE & $\begin{array}{c}\text { PCV in } \\
\text { individual } \\
\text { cattle }\end{array}$ & Mean \pm SE & $\begin{array}{c}\text { PCV in } \\
\text { individual } \\
\text { cattle }\end{array}$ & Mean \pm SE & $\begin{array}{c}\text { PCV in } \\
\text { individual } \\
\text { cattle }\end{array}$ & Mean \pm SE \\
\hline \multirow[t]{15}{*}{$\mathrm{A}$} & 105 & Ivermect & 32.00 & & 32.50 & & 33.50 & & 34.00 & & 35.00 & \\
\hline & 110 & in & 30.50 & & 31.00 & & 31.50 & & 32.50 & & 33.00 & \\
\hline & 115 & $200 \mu \mathrm{g} /$ & 34.00 & & 35.00 & & 36.00 & & 36.00 & & 36.50 & \\
\hline & 120 & $\mathrm{~kg}$ body & 32.50 & & 33.00 & & 33.50 & & 34.00 & & 35,00 & \\
\hline & 125 & weight & 30.00 & & 31.00 & & 32.00 & & 33.50 & & 34.00 & \\
\hline & 130 & & 31.50 & & 32.50 & & 33.00 & & 34.00 & & 35.50 & \\
\hline & 135 & & 35.50 & & 35.00 & & 36.00 & & 36.50 & & 36.50 & \\
\hline & 140 & & 30.50 & $32.23 \pm 0.45$ & 31.00 & $32.83 \pm 0.42$ & 31.50 & $33.62 \pm 0.44$ & 32.00 & $34.36 \pm 0.39$ & 33.00 & $35.06 \pm 0.35$ \\
\hline & 145 & & 32.00 & & 32.50 & & 33.00 & & 34.00 & & 35.00 & \\
\hline & 150 & & 35.50 & & 36.00 & & 36.50 & & 36.50 & & 36.50 & \\
\hline & 155 & & 33.00 & & 33.50 & & 34.00 & & 35.00 & & 35.50 & \\
\hline & 160 & & 30.50 & & 31.00 & & 31.80 & & 32.50 & & 33.00 & \\
\hline & 165 & & 32.50 & & 33.00 & & 33.50 & & 34.00 & & 35.00 & \\
\hline & 170 & & 33.00 & & 33.50 & & 35.00 & & 36.00 & & 36.50 & \\
\hline & 175 & & 31.00 & & 32.00 & & 33.50 & & 35.00 & & 36.00 & \\
\hline \multirow[t]{5}{*}{ B } & 180 & & 32.50 & & 32.00 & & 31.00 & & 30.50 & & 30.00 & \\
\hline & 185 & & 30.00 & & 31.00 & $32.30 \pm 0.70$ & 30.50 & $31.20 \pm 0.52$ & 30.00 & & 29.00 & $29.80 \pm 0.28$ \\
\hline & 190 & Nil & 34.00 & $32.60 \pm 0.99$ & 33.50 & & 32.00 & & 3100 & $30.50 \pm 0.25$ & 30.50 & \\
\hline & 195 & & 35.00 & & 34.00 & & 32.50 & & 31.00 & & 30.00 & \\
\hline & 200 & & 31.50 & & 31.00 & & 33.00 & & 30.00 & & 29.50 & \\
\hline
\end{tabular}

The Erythrocyte Sedimentation Rate (ESR) was decreased significantly at the $14^{\text {th }}, 21^{\text {st }}$ and $28^{\text {th }}$ day of post treatment period in case of the cattle of treatment group but not in case of control group (Table 6).

Table 6. Effect of Ivermectin on Erythrocyte Sedimentation Rate (ESR min/hr) in crossbred cattle

\begin{tabular}{|c|c|c|c|c|c|c|c|c|c|c|c|c|}
\hline \multirow[t]{3}{*}{ Group } & \multirow{3}{*}{$\begin{array}{l}\text { Tag } \\
\text { No }\end{array}$} & \multirow{3}{*}{$\begin{array}{l}\text { Drug with } \\
\text { dose }\end{array}$} & \multicolumn{2}{|c|}{ Pretreatment (Day 0) } & \multicolumn{8}{|c|}{ Post Treatment } \\
\hline & & & \multirow{2}{*}{$\begin{array}{c}\text { ESR in } \\
\text { ind. } \\
\text { cattle }\end{array}$} & \multirow[t]{2}{*}{ Mean \pm SE } & \multicolumn{2}{|c|}{$7^{\text {th }}$ Day } & \multicolumn{2}{|c|}{$14^{\text {th }}$ Day } & \multicolumn{2}{|c|}{$21^{\text {st }}$ Day } & \multicolumn{2}{|c|}{$28^{\text {th }}$ Day } \\
\hline & & & & & $\begin{array}{l}\text { ESR in } \\
\text { ind. cattle }\end{array}$ & Mean \pm SE & $\begin{array}{l}\text { ESR in } \\
\text { ind. cattle }\end{array}$ & Mean \pm SE & $\begin{array}{l}\text { ESR in } \\
\text { ind. cattle }\end{array}$ & Mean \pm SE & $\begin{array}{c}\text { ESR in } \\
\text { ind. cattle }\end{array}$ & Mean \pm SE \\
\hline \multirow[t]{15}{*}{ A } & 105 & & 1.50 & & 1.40 & & 1.30 & & 1.20 & & 1.10 & \\
\hline & 110 & & 0.90 & & 0.80 & & 0.70 & & 0.60 & & 0.60 & \\
\hline & 115 & Ivermectin@ @ & 1.00 & & 0.90 & & 0.80 & & 0.70 & & 0.60 & \\
\hline & 120 & $200 \mu \mathrm{g} / \mathrm{kg}$ & 0.80 & & 0.70 & & 0.60 & & 0.60 & & 0.55 & \\
\hline & 125 & bodyweight & 0.80 & & 0.75 & & 0.70 & & 0.60 & & 0.50 & \\
\hline & 130 & & 1.20 & & 1.00 & & 0.90 & & 0.80 & & 0.70 & \\
\hline & 135 & & 1.10 & $1.13 \pm 0.067$ & 1.00 & $1.03 \pm 0.066$ & 0.85 & $0.93 \pm 0.066$ & 0.80 & $0.85 \pm 0.059$ & 0.65 & $0.77 \pm 0.057$ \\
\hline & 140 & & 1.30 & & 1.20 & & 1.10 & & 1.00 & & 0.90 & \\
\hline & 145 & & 1.40 & & 1.30 & & 1.20 & & 1.10 & & 1.00 & \\
\hline & 150 & & 0.90 & & 0.90 & & 0.80 & & 0.90 & & 0.80 & \\
\hline & 155 & & 0.80 & & 0.70 & & 0.60 & & 0.60 & & 0.50 & \\
\hline & 160 & & 1.10 & & 1.00 & & 0.90 & & 0.80 & & 0.70 & \\
\hline & 165 & & 1.40 & & 1.30 & & 1.20 & & 1.10 & & 1.00 & \\
\hline & 170 & & 1.30 & & 1.20 & & 1.10 & & 1.00 & & 0.90 & \\
\hline & 175 & & 1.50 & & 1.40 & & 1.30 & & 1.20 & & 1.10 & \\
\hline \multirow[t]{5}{*}{$\bar{B}$} & 180 & & 0.90 & & 1.00 & & 1.10 & & 1.20 & & 1.30 & \\
\hline & 185 & & 1.30 & $0.96 \pm 0.104$ & 1.40 & $1.08 \pm 0.216$ & 1.50 & $1.19 \pm 0.097$ & 1.60 & & 1.65 & \\
\hline & 190 & Nil & 0.80 & & 0.90 & & 1.00 & & 1.10 & $1.28 \pm 0.096$ & 1.25 & $1.38 \pm 0.080$ \\
\hline & 195 & & 1.00 & & 1.20 & & 1.20 & & 1.30 & & 1.40 & \\
\hline & 200 & & 0.80 & & 0.90 & & 1.10 & & 1.20 & & 1.30 & \\
\hline
\end{tabular}




\section{Ahammed and others}

\section{The effect of Ivermectin (S/C Formulation) on body weight of crossbred cattle}

Both individual and mean live weight of the cattle of treated group were recorded prior to treatment and on the $28^{\text {th }}$ day of treatment. There was a significant improvement in live weight gain after treatment and it was calculated in kilogram and shown in Table 7.

Table 7. Efficacy of Ivermectin (S/C Formulation) on bodyweight of crossbred cattle

\begin{tabular}{|c|c|c|c|c|c|c|c|}
\hline \multirow[t]{2}{*}{ Group } & \multirow[t]{2}{*}{ Tag no } & \multicolumn{2}{|c|}{ Pretreatment (Day 0) } & \multicolumn{2}{|c|}{ Post treatment (28 ${ }^{\text {th }}$ day) } & \multirow{2}{*}{$\begin{array}{l}\text { Weight gain/ } \\
\text { loss }\end{array}$} & \multirow[t]{2}{*}{ Improvement (\%) } \\
\hline & & body wt (kg) & Mean \pm SE & body wt (kg) & Mean \pm SE & & \\
\hline \multirow[t]{15}{*}{$\mathrm{A}$} & 105 & 110 & \multirow{15}{*}{$144 \pm 7.91$} & 118 & & \multirow{15}{*}{7.4} & \multirow{15}{*}{$5.13 \%$} \\
\hline & 110 & 195 & & 208 & & & \\
\hline & 115 & 120 & & 130 & & & \\
\hline & 120 & 100 & & 106 & & & \\
\hline & 125 & 115 & & 120 & & & \\
\hline & 130 & 150 & & 158 & & & \\
\hline & 135 & 120 & & 126 & & & \\
\hline & 140 & 150 & & 155 & $151.4 \pm 8.16$ & & \\
\hline & 145 & 180 & & 185 & & & \\
\hline & 150 & 140 & & 148 & & & \\
\hline & 155 & 155 & & 158 & & & \\
\hline & 160 & 135 & & 145 & & & \\
\hline & 165 & 130 & & 135 & & & \\
\hline & 170 & 190 & & 198 & & & \\
\hline & 175 & 170 & & 179 & & & \\
\hline \multirow[t]{5}{*}{$\mathrm{B}$} & 180 & 165 & \multirow{5}{*}{$134 \pm 11.37$} & 161 & & \multirow{5}{*}{-3.6} & \multirow{5}{*}{ Nil } \\
\hline & 185 & 120 & & 118 & $130.4 \pm 11.93$ & & \\
\hline & 190 & 150 & & 145 & & & \\
\hline & 195 & 110 & & 108 & & & \\
\hline & 200 & 125 & & 120 & & & \\
\hline
\end{tabular}

In this study subcutaneous injection of Ivermectin was found $100 \%$ effective against gastrointestinal nematodes in crossbred cattle. This effectiveness was determined by fecal egg per gram (EPG) reduction. This result was in conformity with earlier workers (Rehbein et al., 1997; Rendal and Callinan, 1996; Singh et al., 1994; Williams and Plue, 1992; Maru et al., 1990; Soll et al., 1988). Some other workers found more than 99\% efficacy of Ivermectin (Barth et al., 1997; Pitt et al., 1996; Cleartier and Pors, 1994). In this study 100\% efficacy of Ivermectin against ectoparasites were recorded in crossbred cattle. This efficacy was detected by physical examination. Some other workers found 100\% effectiveness of Ivermectin against ectoparasites (Titcherer et al., 1994; Thompson et al., 1994; Genchi and Tada, 1992; Gill et al., 1989). After $7^{\text {th }}$ day to the end of the experiment $\left(28^{\text {th }}\right.$ day post treatment) no ectoparasites were seen on the body of the treated cattle.

The hematological changes in crossbred cattle affected with the gastrointestinal nematode were determined at pre and post treatment period dosed with Ivermectin. The mean value of hemoglobin of the cattle of control group was decreased and this result is in agreement with the reports of Nettleton and Beckelt (1976) and Auosa (1977). In this study, significant increases in $\mathrm{Hb}$ gm\% and PCV\% were found in the treated group of cattle which might be due to expulsion of blood sucking and other parasites from the body of treated cattle. There was a significant reduction in the ESR in treated group of cattle which might be a result of recovery from inflammation in gastrointestinal tract produced by gastrointestinal nematodes. Kumar and Joshi (1992) also found increase in $\mathrm{Hb}$ gm\% level in ivermectin treated cattle.

The body weight of cattle infected with parasites was increased after using anthelmintics. Islam (1992) reported significant $(\mathrm{P}>0.01)$ increase of bodyweight in cows and buffaloes after treatment with anthelmintics. In the present study the live weight gain was 5.13\% after treatment with Ivermectin. This may be a result of proper digestion, absorption and metabolism of feed nutrient because of absence of gastrointestinal nematode infection. In earlier period some workers found improvement of body weight after treatment with Ivermectin (Rehbein et al., 1997; Taylon et al., 1995).

The present study was conducted on a small population of crossbred cattle and the efficacy of Ivermectin was ®determined on the basis of egg count reduction. Since there were some limitations neither any animals were 
slaughtered on post treatment days for detection of presence or absence of nematodes or any milk sample wasexamined for the presence of Ivermectin. It was not possible to study the effect of the drug on pregnant animal and embryo or fetus due to time limitation. Further study should be conducted to observe the effect of the drug on pregnant cattle and its embryo or fetus and its residue in milk.

\section{REFERENCES}

1. Arantes GJ, Sulva CR and Marra DB (1996). Anthelmintic activity of 1\% Ivermectin (injectable solution) in the treatment of calves naturally infected with gastrointestinal nematode. Revista Brasilera and Parasitologia Veterinaria 4: 113-116.

2. Amin MR and Samad MA (1987). Clinico-therapeutic studies on gastrointestinal nematode infection in diarrhoeic cattle. Bangladesh Veterinary Journal 4: 25-28.

3. Ashok K, Yadab CL, Sudesh K and Rajendra K (1996). Experimental evaluation of Ivermectin against gastrointestinal nematodes of sheep. Indian Veterinary Journal 73:782-783.

4. Barth D, Erricsson GF, Kunkle BN, Rehbein S, Ryan WG and Vallace DH (1997). Evaluation of the persistence of the effect of ivermectin and abamectin against gastrointestinal and pulmonary nematodes in cattle. Veterinary Record 140: 278-279.

5. Cleartier C and Pors I (1994). Efficacy of three broad spectrum anthelmintics, against gastrointestinal nematodes in goats. Veterinary Record 134: 523-524.

6. Genchi C and Traldi G (1992). Efficacy of Ivermectin clorsulon combination against gastrointestinal nematodes, Fasciola hepatica and ectoparasites (Psoroptes bovis) in cattle. Atti-della-Societa-Italiana-di-Buiatria 24: 307309.

7. Gill BS, Balakrishnan P, Hossain M and Singh J (1988). Treatment of humpsore-stephanophilariasis of cattle with Ivermectin. Indian Journal of Animal Sciences 58: 552-560.

8. Islam AWMS (1979). Stephanofilariasis in cattle of Bangladesh. Indian Journal of Animal Health 18: 7-8.

9. Kumar A and Joshi BP (1992). Anthelmintic efficacy of ivermectin against naturally occurring gastrointestinal nematodes of sheep. Indian Veterinary Journal 69: 935-937.

10. Maru A, Srivastatava CP and Dubey SC (1990). Efficiency of ivermectin against infection of gastrointestinal nematode in sheep. Indian Journal of Parasitology 14: 241-242.

11. Pitt SR, Heinz-Mutz EM, Baggot DG and Barth D (1996). Control of established infection of parasitic nematodes in cattle by an ivermectin sustained release intraruminal bolus. Helminthologia 33: 125-131.

12. Rehbein S, Pitt SR, Lengholfe WK, Barth D and Eagleson JS (1997). Therapeutic and prophylactic efficacy of Ivomec SR bolus against nematodes and Psoroptes ovis in cattle weighing more than $300 \mathrm{~kg}$ at the time of treatment. Parasitology Research 83: 722-726.

13. Rendal D and Callinan L (1996). The duration of anthelmintics effects of moxidectin and ivermectin in grazing sheep. Australian Veterinary Journal 23: 35.

14. Soulsby EJL (1986). Helminth, Arthropod and Protozoa of domesticated Animals, $7^{\text {th }}$ edition. The ELBS and Baiers, Tindle, Casseil, London, pp.216, 234. 763-766.

15. Sastri UV (1989). Efficacy of Ivermectin against gastrointestinal nematode infection in goat. Indian Veterinary Journal 66: 345-346.

16. Singh J, Gill JS, Ramneck M and Kwatra MS (1994). Comparative efficacy of anthelmintics against nematode infection of sheep and goats in Punjab. Journal of Veterinary Parasitology 8: 47-50.

17. Soll MD, Carmichal IH and Harvey RG (1988). Prophylactic efficacy of sustained release Ivermectin against induced nematode infestation in cattle. Journal of South African Veterinary Association 59: 9-11.

18. Taylon SM, McMullin PF, Mallor TR, Kelly A and Grimashaw WTR (1995). Effects of treatment with tropical ivermectin, three and eight weeks after turnout nematode control and the performance of second beef sucker cattle. Veterinary Records 136: 558-561.

19. Thompson DR, Kugg D, Scott PG, Cramer LG and Barrick RA (1994). Rainfall and breed effects on the efficacy of ivermectin jetting fluid for the prevention of fly strike and treatment of infestation of lice in long wooled sheep. Australian Veterinary Journal 21: 161-164.

20. Titcherer RN, Parry JM and Grimshaw WTR (1994). Efficacy of formulation of abamectin and moxidectin against sucking and biting lice of cattle. Veterinary Record 134: 452-453.

21. Williams JC and Plue RE (1992). Efficacy of Ivermectin delivered from a sustained release bolus against inhibited early fourth stage larvae of Ostertagia ostertagi and other nematodes in cattle. American Journal of Veterinary Research 53: 793-795. 